Canad. Math. Bull. Vol. 29 (1), 1986

\title{
$M$-IDEALS IN $L\left(\ell_{1}, E\right)$
}

\author{
BY
}

\section{J. FLEMING AND D. M. GIARRUSSO}

\begin{abstract}
In this article it is shown that for any Banach space $E$, $L\left(\ell_{1}, E\right)$ always contains uncountably many distinct $M$-ideals that are closed subspaces of $K\left(\ell_{1}, E\right)$ and which are not complemented in $L\left(\ell_{1}, E\right)$. Using standard duality arguments one obtains the result that infinitely many distinct subspaces of $K\left(E, c_{0}\right)$ are $M$-ideals in $L\left(E, c_{0}\right)$. In particular, for the case $E=c_{0}$, this shows that the uniqueness conditions enjoyed by $K\left(\ell_{p}\right)$, $p>1$, is not valid for $E=c_{0}$. The results are obtained by utilizing the identification of $L\left(\ell_{1}, E\right)$ with the vector-valued sequence space $\ell_{x}(E)$ and to exploit natural decompositions of $\ell_{x}(E)^{\prime}$ afforded by a class of $L$ projections on $\ell_{x}(E)^{\prime}$ induced by certain $E^{\prime}$-valued vector measures.
\end{abstract}

1. Introduction. Let $X$ be a Banach space. A continuous linear map $\tau: X \rightarrow X$ is called an $L$-projection if $\tau^{2}=\tau$ and $\|x\|=\|\tau(x)\|+\|x-\tau(x)\|$ for all $x$ in $X$ [4]. A closed subspace $M$ of a Banach space $X$ is an $M$-ideal if there exists an $L$-projection $\tau: X^{\prime} \rightarrow X^{\prime}$ with ker $\tau=M^{\perp} . M$-ideals were first defined and characterized in the fundamental paper of Alfsen and Effros [1].

For Banach spaces $X, Y$ we denote by $L(X, Y)$ [respectively $K(X, Y)$ ] the Banach space of all continuous [respectively compact] linear maps $u: X \rightarrow Y$. Much recent attention has been devoted to the geometric problem concerning the existence and uniqueness of non-trivial $M$-ideals in the operator space $L(X, Y)$. Hennefeld [8] determined that $K\left(\ell_{p}\right), 1<p<\infty$, and $K\left(c_{0}\right)$ are $M$-ideals in $L\left(\ell_{p}\right)$ and $L\left(c_{0}\right)$ respectively. That $K\left(\ell_{2}\right)$ is an $M$-ideals in $L\left(\ell_{2}\right)$ was first established by Dixmier [5]. Recently Flynn [7] has characterized $K\left(\ell_{p}\right)$ as the only non-trivial $M$-ideals in $L\left(\ell_{p}\right), 1<p<\infty$. Saatkamp [10] has shown that $K\left(\ell_{p}, \ell_{q}\right), 1<p \leqq q<\infty$, is an $M$-ideal in $L\left(\ell_{p}, \ell_{q}\right)$ while $K\left(\ell_{1}, \ell_{p}\right), p \geqq 1$, and $K\left(\ell_{p}, \ell_{\infty}\right), 1<p<\infty$, are not $M$-ideals in the corresponding spaces of linear operators. That $K\left(\ell_{1}, C[0,1]\right)$ is not an $M$-ideal in $L\left(\ell_{1}, C[0,1]\right)$ was noted by Mach and Ward [9] who also determined that $K\left(E, c_{0}\right)$ is always an $M$-ideal in $L\left(E, c_{0}\right)$ for any Banach space $E$.

In this paper we show that for any Banach space $E, L\left(\ell_{1}, E\right)$ always contains uncountably many distinct $M$-ideals that are proper closed subspaces of $K\left(\ell_{1}, E\right)$ and which are not complemented in $L\left(\ell_{1}, E\right)$. Using standard duality arguments one obtains the result that infinitely many distinct subspaces of $K\left(E, c_{0}\right)$ (including $K\left(E, c_{0}\right)$ itself) are $M$-ideals in $L\left(E, c_{0}\right)$. In particular for the case $E=c_{0}$ this shows that the uniqueness condition enjoyed by $K\left(\ell_{p}\right), p>1$, is not valid for $K\left(c_{0}\right)$.

Received by the editors August 22, 1983, and, in revised form, October 29, 1984.

AMS Subject Classification: Primary 46B20; Secondary 46A32.

(C) Canadian Mathematical Society 1983. 
The results in this paper are completely self-contained. Our technique is to utilize the identification of $L\left(\ell_{1}, E\right)$ with the vector-valued sequence space $\ell_{\infty}(E)$ and to exploit natural decompositions of $\ell_{\infty}(E)^{\prime}$ afforded by a class of $L$-projections on $\ell_{\infty}(E)^{\prime}$ induced by certain $E^{\prime}$-valued vector measures.

2. Notation and Terminology. Let $E$ be a Banach space over $R$ or $\mathbb{C}$. The vectorvalued sequence space $\ell_{\infty}(E)$ is the collection of all sequences $x=\left(x_{n}\right)$ where $x_{n} \in E$ for all $n$ and $\left(\left\|x_{n}\right\|\right) \in \ell_{\infty}$. Given the norm $\|x\|=\sup _{n}\left\|x_{n}\right\|, \ell_{\infty}(E)$ is a Banach space. The Banach spaces $c_{0}(E)$ and $\ell_{1}(E)$ are similarly defined with norms given by $\sup _{n}\left\|x_{n}\right\|$ and $\Sigma_{n}\left\|x_{n}\right\|$ respectively. $c_{0}(E)$ is a closed subspace of $\ell_{\infty}(E)$.

Let $N$ denote the set of positive integers and $\mathscr{F}$ the power set of $N$. Let $A \in \mathscr{F}, x \in E$ and $\chi_{A}$ the characteristic function of $A$. By $\chi_{A} \cdot x$ we mean the sequence $\left(\chi_{A}(n) x\right)$. The linear span of the collection of all $\chi_{A} \cdot x, A \in \mathscr{F}, x \in E$ will be denoted by $S(E)$. Any such $s \in S(E)$ admits a unique representation $\sum_{i=1}^{n} \chi_{A_{i}} \cdot a_{i}$ where the $a_{i}$ 's are distinct elements of $E$ and $A_{1}, \ldots, A_{n}$ is a disjoint decomposition of $N$. The closure of $S(E)$ in $\ell_{\infty}(E)$ is denoted by $k_{\infty}(E)$. A direct argument shows that $k_{\infty}(E)$ consists of those $\left(x_{n}\right) \in \ell_{\infty}(E)$ such that $\left\{x_{n} \mid n \in N\right\}$ is a relatively compact subset of $E$. Consequently $k_{\infty}(E)$ is always a proper closed subspace of $\ell_{\infty}(E)$ if $\operatorname{dim} E=\infty$.

By $b v a(\mathscr{F}, E)$ we shall mean the collection of all finitely additive $E$-valued set functions $\mu: \mathscr{F} \rightarrow E$ such that

$$
\|\mu\|=\sup \left\{\sum_{i=1}^{n}\left\|\mu\left(A_{i}\right)\right\| \mid n \in N, N=\bigcup_{i=1}^{n} A_{i} \text { (disjoint) }\right\}<\infty
$$

With the above total variation norm $b v a\left(\mathscr{F}_{F}, E\right)$ is a Banach space.

For $A \in \mathscr{F}_{F}$ and $x=\left(x_{n}\right) \in \ell_{\infty}(E)$ we define $\pi_{A}: \ell_{\infty}(E) \rightarrow \ell_{\infty}(E)$ by $\pi_{A}(x)=$ $\left(\chi_{A}(n) x_{n}\right)$. If $A=\{1,2, \ldots, m\}$ we denote $\pi_{A}$ by $\pi_{m}$. The identity map on $E$ is denoted by $i d_{E}$ and ${ }^{t} u: F^{\prime} \rightarrow E^{\prime}$ denotes the transpose of the linear map $u: E \rightarrow F$.

3. $\boldsymbol{L}$-Projections on $\boldsymbol{\ell}_{\infty}(\boldsymbol{E})^{\prime}$. Let $E$ be any Banach space and let $\phi \in \ell_{\infty}(E)^{\prime}$. A finitely additive vector measure $\mu(\phi): \mathscr{F} \rightarrow E^{\prime}$ is constructed as follows: For $A \in \mathscr{F}_{F}$ define the linear form $\mu(\phi)(A)$ on $E$ by

$$
\langle x, \mu(\phi)(A)\rangle=\phi\left(x_{A} \cdot x\right) \quad(x \in E) .
$$

For each $x \in E$

$$
|\langle x, \mu(\phi)(A)\rangle| \leqq\|\phi\|\left\|\chi_{A} \cdot x\right\|_{\infty} \leqq\|\phi\|\|x\|
$$

and hence $\mu(\phi)(A) \in E^{\prime}$ with $\|\mu(\phi)(A)\| \leqq\|\phi\|$. Clearly $\mu(\phi)$ is finitely additive. Further note that $\mu(\phi)=0$ if and only if $\phi$ vanishes on $k_{\infty}(E)$. We now establish that $\mu(\phi)$ is of bounded variation.

3.2 Lemma: Let $E$ be any Banach space and let $\phi \in \ell_{\infty}(E)^{\prime}$. Then $\mu(\phi) \in b v a\left(\mathscr{F}, E^{\prime}\right)$ and $\|\mu(\phi)\|=\left\|\phi \mid k_{\infty}(E)\right\|$.

PROOF: Let $\phi \in \ell_{\infty}(E)^{\prime} ; A_{1}, \ldots, A_{n}$ a partition of $N$ and $x_{1}, \ldots, x_{n}$ elements of $E$ with $\left\|x_{i}\right\| \leqq 1$. Let $\alpha_{i}=\operatorname{sgn}\left\langle x_{i}, \mu(\phi)\left(A_{i}\right)\right\rangle$. Then 


$$
\begin{aligned}
& \sum_{i=1}^{n}\left|\left\langle x_{i}, \mu(\phi)\left(A_{i}\right)\right\rangle\right|=\sum_{i=1}^{n} \alpha_{i}\left\langle x_{i}, \mu(\phi)\left(A_{i}\right)\right\rangle \\
& \quad=\phi\left(\sum_{i=1}^{n} \alpha_{i} \chi_{A_{i}} \cdot x_{i}\right) \leqq\|\phi\|\left\|\sum_{i=1}^{n} \alpha_{i} \chi_{A_{i}} \cdot x_{i}\right\|_{\infty} \leqq\|\phi\|
\end{aligned}
$$

It follows that $\sum_{i=1}^{n}\left\|\mu(\phi)\left(A_{i}\right)\right\| \leqq\|\phi\|$ and so $\|\mu(\phi)\| \leqq\|\phi\|$. Consequently $\mu(\phi) \in b v a\left(\mathscr{F}, E^{\prime}\right)$ for each $\phi \in \ell_{\infty}(E)^{\prime}$.

Now let $\phi \in \ell_{\infty}(E)^{\prime}$. By the Hahn-Banach Theorem $\exists \tilde{\phi} \in \ell_{\infty}(E)^{\prime}$ such that $\tilde{\phi}\left|k_{\infty}(E)=\phi\right| k_{\infty}(E)$ and $\|\tilde{\phi}\|=\left\|\phi \mid k_{\infty}(E)\right\|$. Since $\tilde{\phi}-\phi$ vanishes on $k_{\infty}(E), \mu(\tilde{\phi})=$ $\mu(\phi)$ and so $\|\mu(\phi)\|=\|\mu(\tilde{\phi})\| \leqq\|\tilde{\phi}\|=\left\|k_{\infty}(E)\right\|$. To establish the reverse inequality let $\epsilon>0$ be fixed and choose a simple function $s$ with canonical representation $s=\sum_{i=1}^{n}$ $\chi_{A_{i}} \cdot x_{i}$ where $\left\|x_{i}\right\| \leqq 1$ for $1 \leqq i \leqq n$ and $\left\|\phi\left|k_{\infty}(E) \|-\epsilon \leqq\right| \phi(s) \mid\right.$. Now $|\phi(s)| \leqq \sum_{i=1}^{n}$ $\left|\left\langle x_{i}, \mu(\phi)\left(A_{i}\right)\right\rangle\right| \leqq \sum_{i=1}^{n}\left\|\mu(\phi)\left(A_{i}\right)\right\| \leqq\|\mu(\phi)\|$ and so $\left\|\phi \mid k_{\infty}(E)\right\| \leqq\|\mu(\phi)\|+\epsilon$. It follows that $\|\mu(\phi)\|=\|\phi\| k_{\infty}(E) \|$.

3.3 REMARK: From the above discussion the map $\mu: \ell_{\infty}(E)^{\prime} \rightarrow b v a\left(\mathscr{F}, E^{\prime}\right)$ is continuous and $\operatorname{ker} \mu=k_{\infty}(E)^{\perp}$. For $\omega \in b v a\left(\mathscr{F}, E^{\prime}\right)$ there exists, by the Hahn-Banach Theorem, $\phi_{\omega} \in \ell_{\infty}(E)^{\prime}$ such that

$$
\phi_{\omega}(s)=\sum_{i=1}^{n}\left\langle x_{i}, \omega\left(A_{i}\right)\right\rangle \quad\left(s=\sum_{i=1}^{n} \chi_{A_{i}} \cdot x_{i}\right) .
$$

Consequently $\mu\left(\phi_{\omega}\right)=\omega$ and so the map $\mu$ is surjective. Thus by 3.2 the induced map $\bar{\mu}: \ell_{\infty}(E)^{\prime} / k_{\infty}(E)^{\perp} \rightarrow b v a\left(\mathscr{F}, E^{\prime}\right)$ is an isometric isomorphism and hence $b v a\left(\mathscr{F}, E^{\prime}\right)$ is isometric to the dual space of $k_{\infty}(E)$. In particular $\ell_{\infty}(E)^{\prime}$ is isometrically isomorphic to $b v a\left(\mathscr{F}, E^{\prime}\right)$ if and only if $\operatorname{dim} E<\infty$. If $\operatorname{dim} E=\infty$ then $b v a\left(\mathscr{F}_{F}, E^{\prime}\right)$ captures but a portion of $\ell_{\infty}(E)^{\prime}$ (see section 4).

For $\phi \in \ell_{\infty}(E)^{\prime},\|\mu(\phi)\| \leqq\|\phi\|$ and so, in particular, $\Sigma_{n}\|\mu(\phi)(\{n\})\| \leqq\|\phi\|$. Thus $(\mu(\phi)(\{n\}))_{n}, \in \ell_{1}\left(E^{\prime}\right)$. Now for each $\phi \in \ell_{\infty}(E)^{\prime}$ define the (clearly continuous) linear form $\tau(\phi)$ on $\ell_{\infty}(E)$ by

$$
\tau(\phi)(x)=\Sigma_{n}\left\langle x_{n}, \mu(\phi)(\{n\})\right\rangle \quad\left(x=\left(x_{n}\right) \in \ell_{\infty}(E)\right)
$$

Since

$$
\|\tau(\phi)\|=\Sigma_{n}\|\mu(\phi)(\{n\})\| \leqq\|\phi\| \quad\left(\phi \in \ell_{\infty}(E)^{\prime}\right),
$$

$\tau: \ell_{\infty}(E)^{\prime} \rightarrow \ell_{\infty}(E)^{\prime}$ is continuous. Now $\tau(\phi)(x)=\lim _{m \rightarrow \infty} \phi\left(\pi_{m}(x)\right)$ for each $x \in \ell_{\infty}(E)$ (and consequently $\lim _{m \rightarrow \infty} \phi\left(\left(i d-\pi_{m}\right)(x)\right.$ ) exists for each $x \in \ell_{\infty}(E)$ ). It follows that for each $\phi \in \ell_{\infty}(E)^{\prime}, \tau(\phi)$ agrees with $\phi$ on $c_{0}(E)$ and so ker $\tau=c_{0}(E)^{\perp}$. Since $\mu(\tau(\phi))(A)=\mu(\phi)(A)$ for finite sets $A \in \mathscr{F}$ it is evident that $\tau^{2}=\tau$. We now establish that $\tau$ is an $L$-projection on $\ell_{\infty}(E)^{\prime}$. 
3.5 LemMA: For each $\phi \in \ell_{\infty}(E)^{\prime}$

$$
\|\phi\|=\|\tau(\phi)\|+\|\phi-\tau(\phi)\|
$$

Proof: Since $\Sigma_{n}\|\mu(\phi)(\{n\})\| \leqq\|\phi\|$ it follows that $\sigma_{m}={ }^{t} \pi_{m}$ converges to $\tau$ in the norm topology of $\ell_{\infty}(E)^{\prime}$. Moreover $\pi_{m}$ is an $M$-projection on $\ell_{\infty}(E)$ and hence ${ }^{t} \pi_{m}$ is an $L$-projection on $\ell_{\infty}(E)^{\prime}$. Consequently $\tau$ is an $L$-projection. [see [2], [3]].

The $\tau$-map can now be used to generate a commuting family $\left\{\tau_{A} \mid A \in \mathscr{F}\right\}$ of $L$ projections on $\ell_{\infty}(E)^{\prime}$. For $A \in \mathscr{F}$ let $\sigma_{A}={ }^{\prime} \pi_{A}: \ell_{\infty}(E)^{\prime} \rightarrow \ell_{\infty}(E)^{\prime}$ and define $\tau_{A}=$ $\sigma_{A} \circ \tau$. That is,

$$
\tau_{A}(\phi)(x)=\sum_{n \in A}\left\langle x_{n}, \mu(\phi)(\{n\})\right\rangle \quad\left(x \in \ell_{\infty}(E), \phi \in \ell_{\infty}(E)^{\prime}\right) .
$$

Since $\mu\left(\tau_{A}(\phi)(\{n\})\right)=\chi_{A}(n) \mu(\phi)(\{n\})$ we have

$$
\begin{aligned}
& \tau_{A}\left(\tau_{A}(\phi)(x)\right)=\sum_{n \in A}\left\langle x_{n}, \mu\left(\tau_{A}(\phi)\right)(\{n\})\right\rangle \\
& =\sum_{n \in A}\left\langle x_{n}, \mu(\phi)(\{n\})\right\rangle \\
& =\tau_{A}(\phi)
\end{aligned}
$$

and thus $\tau_{A}^{2}=\tau_{A}$. Furthermore, one easily checks for $A, B \in \mathscr{F}$

[a]

$$
\begin{gathered}
\tau_{A} \circ \tau_{B}=\tau_{A \cap B} \\
\tau_{A \cup B}=\tau_{A}+\tau_{B}-\tau_{A \cap B}
\end{gathered}
$$

[b]

$\sigma_{A}$ is an $L$-projection on $\ell_{\infty}(E)^{\prime}$ since $\pi_{A}$ is an $M$-projection on $\ell_{\infty}(E)$ [1]. Thus

$$
\begin{aligned}
\left\|\tau_{A}(\phi)\right\|+\left\|\phi-\tau_{A}(\phi)\right\| & =\left\|\sigma_{A}(\tau(\phi))\right\|+\| \phi-\sigma_{A}(\tau(\phi) \| \\
& \leqq\left\|\sigma_{A}(\tau(\phi))\right\|+\left\|\tau(\phi)-\sigma_{A}(\tau(\phi))\right\|+\|\phi-\tau(\phi)\| \\
& =\|\tau(\phi)\|+\|\phi-\tau(\phi)\| \\
& =\|\phi\| \quad \text { (by 3.5) }
\end{aligned}
$$

Hence $\left\{\tau_{A}|A \in \mathscr{F}|\right.$ is a family of $L$-projections on $\ell_{\infty}(E)^{\prime}$.

For $A \in \mathscr{F}$ define

$$
c_{A}(E)=\left\{x=\left(x_{n}\right) \in c_{0}(E) \mid x_{n}=0 \text { for } n \notin A\right\} .
$$

Note $c_{N}(E)=c_{0}(E)$.

3.6 Proposition. Let $A \in \mathscr{F}$

[a] $c_{A}(E)$ is an $M$-ideal in $\ell_{\infty}(E)$

[b] If $A$ is infinite then $c_{A}(E)$ is not complemented in $\ell_{\infty}(E)$

[c] $c_{A}(E)^{\prime \prime}$ is isometrically isomorphic to $\ell_{\infty}\left(E^{\prime \prime}\right)$. In particular, if $A$ is infinite, $c_{A}(E)$ is not a dual space. 
Proof: [a] It suffices to note $\operatorname{ker} \tau_{A}=c_{A}(E)^{\perp}$. Let $x \in c_{0}(E), \phi \in \ell_{\infty}(E)^{\prime}$. Then

$$
\begin{aligned}
\phi(x)=\tau(\phi)(x) & =\sum_{n}\left\langle x_{n}, \mu(\phi)(\{n\})\right\rangle \\
& =\sum_{n \in A}\left\langle x_{n}, \mu(\phi)(\{n\})\right\rangle+\sum_{n \in N-A}\left\langle x_{n}, \mu(\phi)(\{n\})\right\rangle .
\end{aligned}
$$

If $\tau_{A}(\phi)=0$ then $\mu(\phi)(\{n\})=0$ for $n \in A$ and hence $\phi(x)=0$ if $x \in c_{A}(E)$. Similarly if $\phi \in c_{A}(E)^{\perp}$ then $\mu(\phi)(\{n\})=0$ for $n \in A$ and so $\tau_{A}(\phi)=0$. Thus $\ell_{\infty}(E)^{\prime}=$ $c_{A}(E)^{\perp} \oplus_{\ell_{1}}$ im $\tau_{A}$.

[b] Let $A \in \mathscr{F}$ be infinite and suppose there exists a continuous projection $\xi$ on $\ell_{\infty}(E)$ with $\operatorname{im} \xi=c_{A}(E)$. Let $x_{0} \in E, x_{0}^{\prime} \in E^{\prime}$ be such that $\left\langle x_{0}, x_{0}^{\prime}\right\rangle=1$ and let $\iota: N \rightarrow A$ be the natural order preserving bijection. Denote by $\hat{\imath}$ the induced embedding of $\ell_{\infty}$ into $\ell_{\infty}(E)$. That is, for $\beta \in \ell_{\infty}$,

$$
\hat{\imath}(\beta)(n)=\beta_{\iota^{-1}(n)} x_{\mathrm{o}} \text { if } n \in A, \quad \hat{\imath}(\beta)(n)=0 \text { if } n \notin A
$$

Finally let $\bar{p}$ be the mapping from $c_{A}(E)$ into $c_{0}$ given by

$$
\bar{p}(x)=\left(\left\langle p_{\iota(n)}(x), x_{0}^{\prime}\right\rangle\right)_{n} \quad\left(x \in c_{A}(E)\right)
$$

where $p_{n}: \ell_{\infty}(E) \rightarrow E$ is the $n$-th coordinate projection map on $\ell_{\infty}(E)$. Note that $\hat{\imath}(\beta) \in c_{A}(E)$ if $\beta \in c_{0}$. Consequently, for $\beta \in c_{0}, \bar{p}_{\circ} \xi \circ \hat{\imath}(\beta)=\bar{p}(\xi(\hat{\imath}(\beta)))=\bar{p}(\hat{\imath}(\beta))$ $=\left(\left\langle p_{\iota(n)}(\hat{\imath}(\beta)), x_{0}^{\prime}\right\rangle\right)_{n}=\left(\left\langle\beta_{n} x_{0}, x_{0}^{\prime}\right\rangle\right)_{n}=\beta$. Thus $\bar{p}_{\circ} \xi_{\circ} \hat{\imath}$ gives a continuous projection of $\ell_{\infty}$ onto $c_{0}$ which is impossible.

[c] if $A$ is infinite then $c_{A}(E)$ is isometrically isomorphic to $c_{0}(E)$. The result follows by standard duality arguments.

For any Banach space $E, L\left(\ell_{1}, E\right)$ is isometrically isomorphic to $\ell_{\infty}(E)$ under the isometry $\rho_{E}$ given by $\rho_{E}(u)=\left(u\left(e_{n}\right)\right)_{n}$. One directly verifies that $\rho_{E}\left(K\left(\ell_{1}, E\right)\right)=$ $k_{\infty}(E)$. For each $A \in \mathscr{F}$ we define $K_{A}\left(\ell_{1}, E\right)=p_{E}^{-1}\left(c_{A}(E)\right)$. Note that $K_{A}\left(\ell_{1}, E\right)$ is always a proper closed subspace of $K\left(\ell_{1}, E\right)$. From 3.6 we have

\subsection{COROLlary: Let $E$ be any Banach space and let $A \in \mathscr{F}_{F}$}

[a] $K_{A}\left(\ell_{1}, E\right)$ is an M-ideal in $L\left(\ell_{1}, E\right)$

[b] $K_{A}\left(\ell_{1}, E\right)$ is not complemented in $L\left(\ell_{1}, E\right)$ if $A$ is infinite

[c] $K_{A}\left(\ell_{1}, E\right)^{\prime \prime}$ is isometrically isomorphic to $L\left(\ell_{1}, E^{\prime \prime}\right)$ if $A$ is infinite.

Now using the transpose map $t: L\left(E, c_{0}\right) \rightarrow L\left(\ell_{1}, E^{\prime}\right)$ and corollary 3.7 we obtain a family of $M$-ideals in $L\left(E, c_{0}\right)$ by pulling back the $M$-ideals $K_{A}\left(\ell_{1}, E^{\prime}\right)$. We begin with an elementary lemma.

3.8 Lemma: Let $E$ be any Banach space and let $t: L\left(E, c_{0}\right) \rightarrow L\left(\ell_{1}, E^{\prime}\right)$ denote the transpose map. Then $t\left(K\left(E, c_{0}\right)=K_{N}\left(\ell_{1}, E^{\prime}\right)\right.$.

Proof: Let $u \in K_{N}\left(\ell_{1}, E^{\prime}\right)$ and define $v: E \rightarrow c_{0}$ by $v(x)=\left(\left\langle x, u\left(e_{n}\right)\right\rangle\right)$. Clearly $v$ is continuous and for $x \in E,\left\langle x,{ }^{t} v\left(e_{n}\right)\right\rangle=\left\langle v(x), e_{n}\right\rangle=\left\langle x, u\left(e_{n}\right)\right\rangle$. Thus ${ }^{t} v=u$. Moreover $v \in K\left(E, c_{0}\right)$ since $u$ is compact. 
Now for $x^{\prime} \in E^{\prime}, \beta \in c_{0}$ let $w: E \rightarrow c_{0}$ denote the rank-1 operator $w(x)=\left\langle x, x^{\prime}\right\rangle \beta$. For each $x \in E, n \in N\left\langle x,{ }^{t} w\left(e_{n}\right)\right\rangle=\left\langle w(x), e_{n}\right\rangle=\beta_{n}\left\langle x, x^{\prime}\right\rangle$. Thus $\left\|{ }^{t} w\left(e_{n}\right)\right\| \leqq\left\|x^{\prime}\right\|\left|\beta_{n}\right|$ and consequently ${ }^{t} w \in K_{N}\left(\ell_{1}, E^{\prime}\right)$. Since $K\left(E, c_{0}\right)$ is the closed linear span of rank-1 maps it follows that ${ }^{t} u \in K_{N}\left(\ell_{1}, E^{\prime}\right)$ for each $u \in K\left(E, c_{0}\right)$.

Let $Z$ be a Banach space, $M, Y$ closed subspaces such that $M \subset Y \subset Z$ and suppose $M$ is an $M$-ideal in $Z$. Let $\tau$ denote the $L$-projection on $Z^{\prime}$ with kernel $M^{\perp}$. For $\phi \in Y^{\prime}$ let $\bar{\phi}$ be any continuous linear extension of $\phi$ to all of $Z$ and define $\bar{\tau}(\phi)=\tau(\bar{\phi}) \mid Y$. It is easy to see that $\bar{\tau}: Y^{\prime} \rightarrow Y^{\prime}$ is an $L$-projection on $Y^{\prime}$ with $\operatorname{ker} \bar{\tau}=M^{\perp}$ (in $Y^{\prime}$ ).

3.9 Corollary: For each $A \in F_{F}$ let $M_{A}\left(E, c_{0}\right)=t^{-1}\left(K_{A}\left(\ell_{1}, E^{\prime}\right)\right)$. Then $M_{A}\left(E, c_{0}\right)$ is an $M$-ideal in $L\left(E, c_{0}\right)$ and if $A$ is infinite, $M_{A}\left(E, c_{0}\right)^{\prime \prime}$ is isometrically isomorphic to $L\left(\ell_{1}, E^{\prime \prime \prime}\right)$.

Proof: Let $t: L\left(E, c_{0}\right) \rightarrow L\left(\ell_{1}, E\right)$ be the transpose map. By 3.8 we have $t\left(M_{A}\left(E, c_{0}\right)\right)$ $=K_{A}\left(\ell_{1}, E^{\prime}\right)$ and $K_{A}\left(\ell_{1}, E^{\prime}\right) \subset t\left(L\left(E, c_{0}\right)\right) \subset L\left(\ell_{1}, E^{\prime}\right)$. By $3.7 K_{A}\left(\ell_{1}, E^{\prime}\right)$ is an $M$-ideal in $L\left(\ell_{1}, E^{\prime}\right)$ and so by the above discussion $K_{A}\left(\ell_{1}, E^{\prime}\right)$ is an $M$-ideal in $t\left(L\left(E, c_{0}\right)\right)$. Since $t$ is an isometry onto its image it follows that $M_{A}\left(E, c_{0}\right)=t^{-1}\left(K_{A}\left(\ell_{1}, E^{\prime}\right)\right)$ is an $M$-ideal in $L\left(E, c_{0}\right)$.

3.10 REMARK: It is of interest to unravel the identifications and exhibit explicitly the form of the $L$-projections on $L\left(E, c_{0}\right)^{\prime}$ with kernel $M_{A}\left(E, c_{0}\right)^{\perp}$.

We have $t: L\left(E, c_{0}\right) \rightarrow L\left(\ell_{1}, E^{\prime}\right)$ (isometry into) and $\rho_{E^{\prime}}: L\left(\ell_{1}, E^{\prime}\right) \rightarrow \ell_{\infty}\left(E^{\prime}\right)$ (isometry onto). We have the following where $\gamma=\rho_{E^{\prime}}$ ot

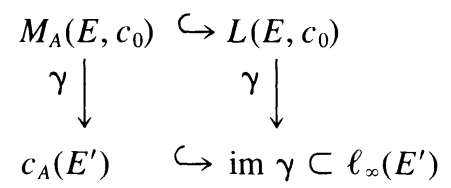

Let $\tau_{A}$ be the $L$-projection on $\ell_{\infty}\left(E^{\prime}\right)^{\prime}$ with $\operatorname{ker} \tau_{A}=c_{A}\left(E^{\prime}\right)^{\perp}$ and $\bar{\tau}_{A}$ the induced $L$-projection on $(\operatorname{im} \gamma)^{\prime}$ with $\operatorname{ker} \bar{\tau}_{A}=c_{A}\left(E^{\prime}\right)^{\perp}$ (in $\left.(\operatorname{im} \gamma)^{\prime}\right)$. The $L$-projection on $L\left(E, c_{0}\right)^{\prime}$ (with kernel $M_{A}\left(E, c_{0}\right)^{\perp}$ ) whose structure we wish to unravel is precisely ${ }^{t} \gamma \circ \bar{\tau}_{A} \circ{ }^{t} \gamma^{-1}$.

A direct computation shows that

$$
{ }^{t} \gamma \circ \bar{\tau}_{A} \circ \gamma^{-1}(\phi)(u)=\sum_{n \in A} \phi\left(\left(e_{n} \otimes e_{n}^{\prime}\right) \circ u\right)
$$

REMARK: Corollary 3.9 in the case $A=N$ was first established by Mach and Ward [9] using the 3-balls-property. A different proof was given by Saatkamp in [10].

4. Further Remarks on the Dual of $\boldsymbol{\ell}_{\infty}(E)$. In a recent paper [6] the authors have determined certain natural topological decompositions of the strong dual of $L_{b}(Z, E)$ where $Z, E$ are Hausdorff locally convex spaces and $L_{b}(Z, E)$ carries the topology of uniform convergence on the bounded subsets of $Z$. It is shown that if $E$ is quasicomplete and $\mathrm{id}_{E}$ has a suitable resolution into quasi-compact maps then $K_{b}(Z, E)^{\perp}$ is topologically complemented in $L_{b}(Z, E)^{\prime}$ where $K_{b}(Z, E)$ is the space of continuous 
linear maps $u: Z \rightarrow E$ which take bounded sets to relatively compact sets (such maps are called quasi-compact). $K_{b}(Z, E)$ is always a closed subspace of $L_{b}(Z, E)$ whenever $E$ is quasi-complete, and $K_{b}(Z, E)$ coincides with the space of compact linear maps when $Z, E$ are Banach spaces. It follows that for any Banach space $Z$ and a large class of Banach spaces $E$ [see definition 4.1] $K(Z, E)^{\perp}$ is complemented in $L(Z, E)^{\prime}$. In particular $K\left(\ell_{1}, E\right)^{\perp}$ is topologically complemented in $L\left(\ell_{1}, E\right)^{\prime}$ and $k_{\infty}(E)^{\perp}$ is complemented in $\ell_{\infty}(E)^{\prime}$. In this section we explicitly construct projections on $\ell_{\infty}(E)^{\prime}$ with kernels $k_{\infty}(E)^{\perp}$ and study the relationship between these projections and the $L$ projection $\tau$ on $\ell_{\infty}(E)^{\prime}$ defined in section 3 .

For simplicity we make the following definition

4.1 Definition: A Banach space $E$ is admissible if there exists a sequence of compact operators $\zeta_{n}: E \rightarrow E$ such that

$$
x=\Sigma_{n} \zeta_{n}(x) \quad(x \in E)
$$

and

$$
\sup \left\{\left\|\sum_{n=1}^{m} \beta_{n} \zeta_{n}\right\| \mid \beta=\left(\beta_{n}\right) \in \ell_{\infty},\|\beta\|_{\infty} \leqq 1, m \geqq 1\right\}<\infty .
$$

Examples of admissible Banach spaces are afforded by Banach spaces of the type $\left(\Sigma_{n} \otimes X_{n}\right)_{\ell_{p}}, p \geqq 1$ or $\left(\Sigma_{n} \otimes X_{n}\right)_{c_{0}}$ where $\operatorname{dim} X_{n}<\infty$.

Let $E$ be an admissible Banach space. Define $\chi: \ell_{\infty}(E)^{\prime} \rightarrow \ell_{\infty}(E)^{\prime}$ by

$$
\chi(\phi)(x)=\Sigma_{n} \phi\left(\zeta_{n} x_{1}, \zeta_{n} x_{2}, \ldots\right) \quad\left(x \in \ell_{\infty}(E), \phi \in \ell_{\infty}(E)^{\prime}\right) .
$$

From 4.1.2 it follows that the series in 4.2 is absolutely convergent and in turn that $\chi$ is a continuous operator on $\ell_{\infty}(E)^{\prime}$. A direct computation shows that for $\phi \in \ell_{\infty}(E)^{\prime}$, $\mu(\chi(\phi))=\mu(\phi)$ and hence $\phi\left|k_{\infty}(E)=\chi(\phi)\right| k_{\infty}(E)$. Thus $\chi^{2}=\chi$ and ker $\chi=k_{\infty}(E)^{\perp}$. Furthermore $\tau \circ \chi=\chi \circ \tau=\tau$ where $\tau$ is the $L$-projection defined in section 3. Consequently $\tau, \chi-\tau$ and id $-\chi$ are mutually orthogonal projections on $\ell_{\infty}(E)^{\prime}$ and thus we obtain the topological decomposition

$$
\ell_{\infty}(E)^{\prime}=\operatorname{ker}(\mathrm{id}-\tau) \oplus \operatorname{ker}(\mathrm{id}-\chi+\tau) \oplus \operatorname{ker} \chi
$$

where ker (id $-\tau)$ is isometrically isomorphic to $\ell_{1}\left(E^{\prime}\right)$ and $\operatorname{ker}(\mathrm{id}-\chi+\tau)$ is isometrically isomorphic to $b v a_{0}\left(\mathscr{F}, E^{\prime}\right)$, the space of $E^{\prime}$-valued vector measures which vanish on the finite subsets of $N$. Note that card $b v a_{0}\left(\mathscr{F}^{\prime}, E^{\prime}\right) \geqq 2^{c}$.

From $3.5 \tau$ is always an $L$-projection. If $\chi$ is also an $L$-projection then for each $\phi \in \ell_{\infty}(E)^{\prime}$

$$
\begin{aligned}
\|\phi\| & =\|\chi(\phi)\|+\|\phi-\chi(\phi)\| \\
& =\|\tau(\chi(\phi))\|+\|\chi(\phi)-\tau(\chi(\phi))\|+\|\phi-\chi(\phi)\| \\
& =\|\tau(\phi)\|+\|\chi(\phi)-\tau(\phi)\|+\|\phi-\chi(\phi)\|
\end{aligned}
$$


If $E=c_{0}$ then $\chi$ is an $L$-projection. This follows from the direct calculation ${ }^{t} \rho_{c_{0}}^{-1} \circ \sigma \circ o^{t} \rho_{c_{0}}$ $=\chi$ where $\rho_{c_{0}}$ is the isometry of $L\left(\ell_{1}, c_{0}\right)$ onto $\ell_{\infty}\left(c_{0}\right)$ and $\sigma={ }^{t} \gamma_{\circ} \bar{\tau}_{\circ}{ }^{t} \gamma^{-1}$ with $\gamma$ and $\bar{\tau}$ defined as in section 3 (with $E=\ell_{1}$ ). Consequently, in this case we have the interesting decomposition

$$
\ell_{\infty}\left(c_{0}\right)^{\prime}=\operatorname{im} \tau \oplus \ell_{1} \operatorname{im}(\chi-\tau) \oplus \ell_{\ell_{1}} k_{\infty}\left(c_{0}\right)^{\perp}
$$

where im $\tau$ is isometrically isomorphic to $\ell_{1}\left(\ell_{1}\right)$ and $\operatorname{im} \tau \oplus \operatorname{im}(\chi-\tau)$ is isometrically isomorphic to $b v a\left(\mathscr{F}_{,}, \ell_{1}\right)$ which is in turn isometrically isomorphic to $\ell_{1}\left(\ell_{\infty}^{\prime}\right)$.

The functionals in $k_{\infty}\left(c_{0}\right)^{\perp}$ are of a more exotic nature than those in $b v a\left(\mathscr{F}, \ell_{1}\right)$. To obtain examples of such functionals let $\eta$, $\xi$ be elements of $\ell_{\infty}^{\prime}$ where $\eta$ extends the limit functional on $c$ and $\xi \in c_{0}^{\perp}$. Then $\xi \otimes \eta \in \ell_{\infty}\left(\ell_{\infty}\right)^{\prime}$ where $\xi \otimes \eta(x)=\xi\left(\left(\left\langle x_{n}, \eta\right\rangle\right)_{n}\right)$ for $x=\left(x_{n}\right) \in \ell_{\infty}\left(\ell_{\infty}\right)$. Clearly $\xi \otimes \eta$ vanishes on $c_{0}\left(\ell_{\infty}\right)$. Let

$$
\pi=\rho_{\ell_{x}} \circ t \circ \rho_{c_{0}}^{-1}: \ell_{\infty}\left(c_{0}\right) \rightarrow \ell_{\infty}\left(\ell_{\infty}\right)
$$

where $t: L\left(\ell_{1}, c_{0}\right) \rightarrow L\left(\ell_{1}, \ell_{\infty}\right)$ is the transpose map and $\rho_{c_{0}}: L\left(\ell_{1}, c_{0}\right) \rightarrow \ell_{\infty}\left(c_{0}\right)$, $\rho_{\ell_{\infty}}: L\left(\ell_{1}, \ell_{\infty}\right) \rightarrow \ell_{\infty}\left(\ell_{\infty}\right)$ are the canonical isometries. From $3.8 \pi\left(k_{\infty}\left(c_{0}\right)\right)=c_{0}\left(\ell_{\infty}\right)$ and hence $\xi \otimes \eta \circ \pi \in k_{\infty}\left(c_{0}\right)^{\perp}$. Moreover the map $\xi \rightarrow \xi \otimes \eta \circ \pi$ is injective for if $\beta \in \ell_{\infty}$ and if $x_{n}=\sum_{i=1}^{n} \beta_{i} e_{i}$ for each $n$ then $\xi \otimes \eta \circ \pi\left(\left(x_{n}\right)\right)=\xi(\beta)$. It follows that card $k_{\infty}\left(c_{0}\right)^{\perp} \geqq \operatorname{card} c_{0}^{\perp}=2^{c}$.

REMARKS: Let $j_{x}\left(c_{0}\right)$ denote the closed linear span of all forms of the type $\xi \otimes \eta \circ \pi$, $\xi \in c_{0}^{\perp}$ and $\eta \in \ell_{\infty}^{\prime}$ extending the limit functional. It would be of interest to describe the quotient space $k_{\infty}\left(c_{0}\right)^{\perp} / j_{\infty}\left(c_{0}\right)$.

\section{REFERENCES}

1. Alfsen, E. M. and Effros, E. G., Structure in real Banach spaces I, Ann. of Math., 96 (1972), pp. $98-173$.

2. Behrends, E., et al., $L^{\prime \prime}$ structure in real Banach spaces, 613, Springer Lecture Notes.

3. Behrends, E., "M structure and the Banach Stone Theorem", 736, Springer Lecture Notes.

4. Cunningham, F., L-structures in L-spaces, Trans. Amer. Math. Soc., 95 (1960), pp. 274-299.

5. Dixmier, J, Les fonctionnelles linéaires sur l'ensembles des opérateurs bornés d' un espace de Hilbert, Ann. of Math, 51 (1950), pp. 387-408.

6. Fleming, D. J. and Giarrusso, D. M., Topological decompositions of the duals of locally convex operator spaces, Math. Proc. Camb. Phil. Soc., 93 (1983), pp. 307-314.

7. Flynn, P., A characterization of $M$-ideals in $B\left(\ell_{p}\right)$ for $l<p<\infty$, Pac. J. Math, 98 (1982), pp. 73-80.

8. Hennefeld, J. A., A decomposition for $B(X)^{*}$ and unique Hahn-Banach extensions, Pac. J. Math, 46 (1973), pp. 197-199.

9. Mach, J. and Ward, J., Approximation by compact operators on certain Banach spaces, J. Approximation Theory, 23 (1978), pp. 274-286.

10. Saatkamp, K., M-ideals of compact operators, Math. Z., 158 (1978), pp. 253-263.

Department of Mathematics

ST. LAWRENCE UNIVERSITY

CANTON, NY 13617

U.S.A. 